\title{
滇西北高山微水体与溪流生境底栖 动物多样性和环境特征
}

\author{
刘硕然 ${ }^{1,2,3}$ 杨道德 $1^{*}$ 李先福 $2,3,4$ 谭 路 ${ }^{4}$ 孙 军 $^{5}$ 和晓阳 ${ }^{5}$ 杨文书 ${ }^{5}$ \\ 任国鹏 2,3 Davide Fornacca ${ }^{2,3}$ 蔡庆华 ${ }^{4}$ 肖 文 $^{2,3^{*}}$ \\ 1 (中南林业科技大学野生动植物保护研究所, 长沙 410004) \\ 2 (大理大学东喜玛拉雅研究院, 云南大理 671003) \\ 3 (中国三江并流区域生物多样性协同创新中心, 云南大理 671003) \\ 4 (中国科学院水生生物研究所淡水生态与生物技术国家重点实验室, 武汉 430072) \\ 5 (高黎贡山国家级自然保护区贡山管理局, 云南怒江 673500)
}

摘要: 高山微水体由于面积微小且通过地表径流形成串联结构常常被认为与高山溪流具有类似的生境, 然而由于 这两类生境中环境因子与底栖动物多样性存在差异, 它们在生态系统中的作用可能完全不同。滇西北地区是全球 生物多样性热点区域之一, 境内高山微水体和高山溪流分布密集, 在区域底栖生物多样性维持方面具有重要的功 能, 然而目前对这两类高山淡水生态系统的研究较少。为了比较这两类生境环境因子的异同及其对底栖动物多样 性的维持作用, 2015年6月，作者在云南省怒江州贡山县的高山峡谷内，对 27 个高山微水体和同区域分布的1条高 山溪流(海拔高差500 m范围)的底栖动物多样性和水环境因子进行了实地调查。结果表明: (1)高山微水体和高山溪 流底栖动物群落中优势分类单元种群数量均比较庞大, 而稀有分类单元数量较多且种群较小; (2)两种生境在环境 因子、物种多样性、功能多样性和群落结构方面的差异明显, 高山溪流有较高的物种丰富度、物种多样性和功能 多样性; (3)高山微水体底栖动物多样性的分布与水环境因子无关, 而高山溪流底栖动物多样性与群落结构的形成 受到与流速关联的水环境因子和海拔的影响。因此, 高山微水体与高山溪流不能简单地视为类似的生境类型, 它 们对区域底栖动物多样性和生态功能维持可能具有不同的作用。

关键词: 高山淡水生态系统; 环境异质性; 群落结构; 生物多样性维持; 高黎贡山

\section{Diversity in benthic and environmental characteristics on alpine micro-waterbodies and stream ecosystems in northwest Yunnan}

\footnotetext{
Shuoran $\mathrm{Liu}^{1,2,3}$, Daode Yang ${ }^{1 *}$, Xianfu $\mathrm{Li}^{2,3,4}$, Lu Tan ${ }^{4}$, Jun Sun ${ }^{5}$, Xiaoyang $\mathrm{He}^{5}$, Wenshu Yang ${ }^{5}$, Guopeng $\mathrm{Ren}^{2,3}$, Davide Fornacca ${ }^{2,3}$, Qinghua Cai ${ }^{4}$, Wen Xiao ${ }^{2,3^{*}}$

1 Institute of Wildlife Conservation, Central South University of Forestry and Technology, Changsha 410004

2 Institute of Eastern Himalaya Biodiversity Research, Dali University, Dali, Yunnan 671003

3 Collaborative Innovation Center for Biodiversity and Conservation in the Three Parallel Rivers Region of China, Dali, Yunnan 671003

4 State Key Laboratory of Freshwater Ecology and Biotechnology, Institute of Hydrobiology, Chinese Academy of Sciences, Wuhan 430072

5 Gongshan Administration Bureau, Gaoligong Mountain National Nature Reserve, Nujiang, Yunnan 673500
}

\begin{abstract}
Due to the small area and the cascaded structure formed by surface water, alpine micro-waterbodies are often considered to have similar habitats to alpine streams. However, due to the differences between the environmental factors and the benthic diversity, the functions of these two habitats in the ecosystem may be completely different. Northwest Yunnan hosts one of the richest global biodiversity
\end{abstract}


hotspots where alpine micro-waterbodies and streams are densely concentrated. These two fresh water ecosystems have important functions in regional benthic biodiversity maintenance, however, these peculiar freshwater ecosystems have barely received research attention. In order to compare the similarities and differences of environmental factors and biodiversity between these two habitats and their maintenance effects on benthic diversity, in this study, benthic biodiversity and the environmental factors of 27 alpine micro-waterbodies and a stream in the same region (9 sample sites within an altitude gradient of $500 \mathrm{~m}$ ) were investigated in an alpine valley of Gongshan County, Nujiang, Yunnan Province in June of 2015. Results showed that: (1) The common characteristics of benthic communities in alpine micro-waterbodies and the stream were that the dominant taxa have large population size, while, the rare taxa had higher richness but small population size. (2) However, the environmental factors, species diversity, functional diversity and community structures were quite different between the alpine micro-waterbodies and the stream, the alpine stream had higher species richness, species diversity and functional diversity than alpine micro-waterbodies. (3) the benthic biodiversity and formation of community structure in alpine stream were related to the elevation and aquatic environmental factors relating to flow rate regulation, while, the aquatic environmental factors of alpine micro-waterbodies did not act as the drivers for the distribution of benthic biodiversity. Therefore, these findings suggest that alpine micro-waterbodies and streams are distinct ecosystems that each feature has very different characteristics, they cannot be regarded as similar ecosystem types. Both of them play an important role in the maintenance of regional benthic biodiversity and ecosystem functions.

Key words: alpine freshwater ecosystem; environmental heterogeneity; community structure; biodiversity maintenance; Gaoligong Mountain

生物多样性的研究重点是人类社会经济发展 与生物多样性丧失之间的矛盾(Myers, 1988; Myers et al, 2000)。淡水生态系统相对脆弱, 遭到破坏后更 难恢复, 其生物多样性的丧失比陆地生态系统更为 严重(Sala et al, 2000), 故其生物多样性的保护面临 着更大的挑战(Dudgeon et al, 2006)。

滇西北地处中国西南山地、喜马拉雅及南亚次 大陆三个全球生物多样性热点区的交汇区(Hoffman et al, 2016), 生物多样性资源十分丰富。该地区海拔 较高处有一类特殊的天然水体, 与溪流和河流相比, 其边界形态相对封闭、面积微小、数量众多, 是滇 西北最常见但又是最容易受到忽视的淡水生态系 统。刘硕然等(2017)将这类天然水体命名为微水体 (micro-waterbodies)。微水体之间可通过高山溪流形 成相互联系的完整系统, 具有为上下游来回迁移的 水生生物提供栖息地等重要的生态功能(Stokstad, 2014)。

高山微水体因面积微小, 常常被视为高山溪流 生境类型, 但实际情况可能并非如此。第一, 高山 溪流滞水区和激流区之间的水文特征虽有差异(Taft \& Koncelik, 2006; Belletti et al, 2015), 但高山微水 体与高山溪流的底质及水文特征差异更加显著, 相 关的水环境指标可能截然不同, 导致这两类生境水 生生物群落同样存在显著差异(Miserendino, 2001;
Bazzanti et al, 2003)。第二, 相邻或距离非常近的两 个微水体之间能产生较高的环境异质性(Liu et al, 2018), 这种现象在高山溪流中一般不会发生。第三, 由干流与支流形成的复杂河流网络生态系统, 其水 环境和生物群落明显地受到连通性的影响(Carrara et al, 2012, 2014; Altermatt, 2013), 而微水体系统之 间可依赖溪流支流连通, 最终汇集并输出到高山溪 流干流中, 故微水体生境中的物质和生物可能会影 响高山溪流的水环境及生物群落。底栖动物作为高 山微水体和高山溪流最优势的生物类群, 充分代表 了这两类生境的生物多样性特征。

为比较高山微水体与高山溪流在环境因子、水 生生物多样性和群落结构上的异同, 分析环境因子 对生物多样性分布格局的影响, 探讨这两种生境对 区域底栖动物多样性的维持作用, 我们在云南省怒 江州贡山县的一片高山峡谷, 对同域分布的高山微 水体与高山溪流生态系统的环境异质性和底栖动 物多样性进行了实地调查。

\section{研究方法}

\section{1 研究区域}

野外研究地点位于云南省怒江州贡山县, 属怒 江流域, 地处滇西北高山峡谷区, 大小河流众多、 地势险陡、雨量充沛，水利资源极为丰富。本地区 
天然植被以松(Pinus)、杉类针叶林为主, 辅以箭竹 (Fargesia) 类草本植物。

研究区域位于贡山县城至贡山县独龙江乡废 弃公路途中的一片高山峡谷湿地, 地理位置为 98 $27^{\prime} 33^{\prime \prime}-98^{\circ} 28^{\prime} 09^{\prime \prime}$ E, $27^{\circ} 4750^{\prime \prime}-27^{\circ} 46^{\prime} 33^{\prime \prime} \mathrm{N}$, 海 拔3,087-3,586 m, 整个区域面积约 $1.4 \mathrm{~km}^{2}$ 。年平均 气温 $7.7^{\circ} \mathrm{C}$, 年平均降水量 $2,525 \mathrm{~mm}$ (根据贡山县林 业局安装在县城至独龙江乡老公路与新公路交叉
口处的全自动野外实时监测气象站2014年8月1日 至2015年7月31日的数据计算)。为方便起见，将研 究区域内的微水体群定义为A区, 溪流为AS (图1)。 在A区内，上述废弃的老公路将整个微水体群分割 成两个小的微水体群 $\mathrm{AD}$ 和 $\mathrm{AU}$ ，两者之间水体没有 直接连通。位于微水体群西边的溪流(AS), 沿山谷 坡势顺流而下，无径流穿越微水体群; 微水体群则 有径流汇入溪流形成连通。

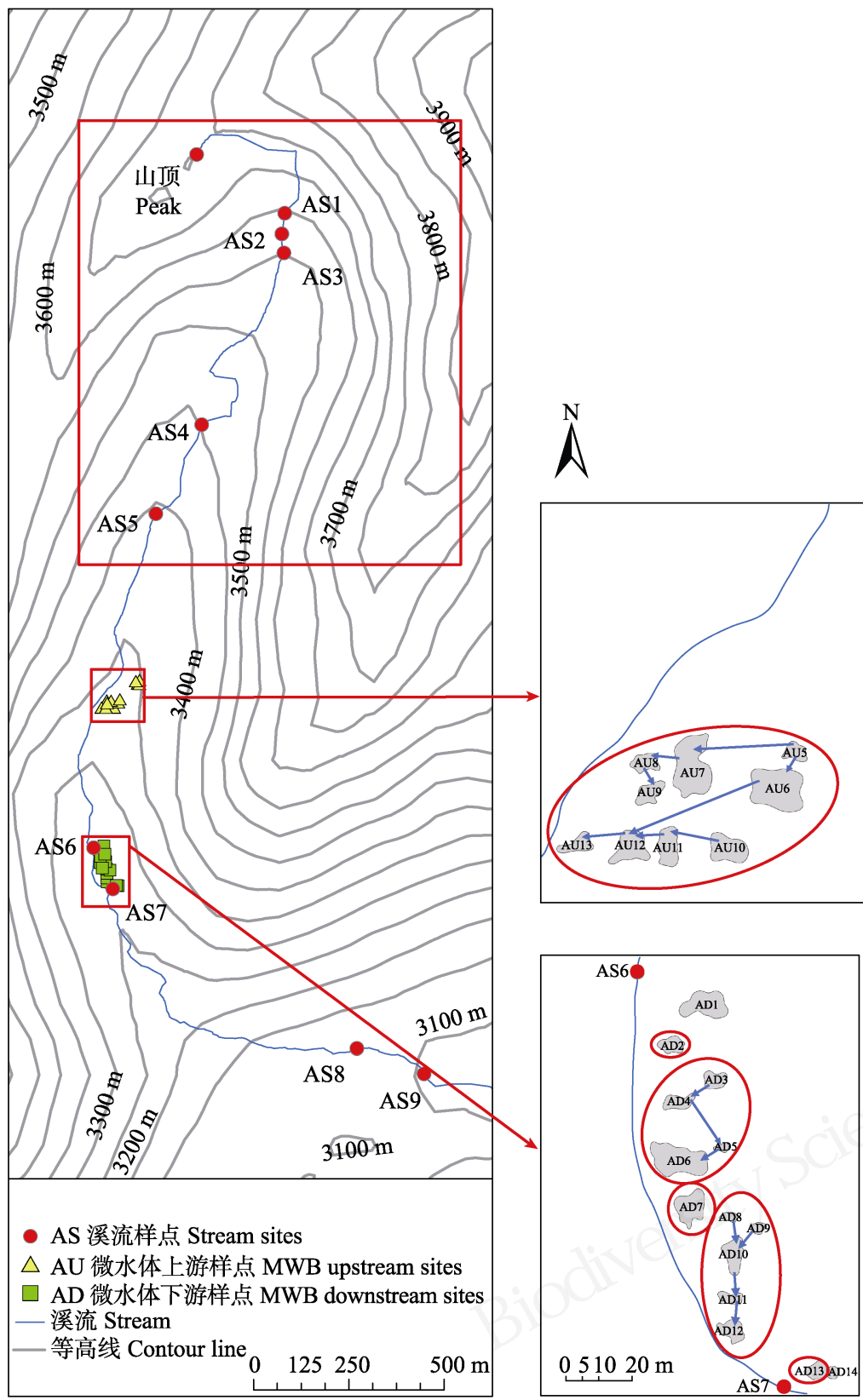

图1 研究区域及样点图示(微水体串联图示中圈内的微水体串最终与溪流连通)

Fig. 1 Research area and sampling sites. The micro-waterbodies (MWB) which are circled indicate that the water flow from the micro-waterbody cascades is running into the stream finally. 


\section{2 采样和鉴定}

由于所有微水体每年均经历 5 个月以上的季节 性封冻期(一般从11月至翌年4月), 故调查取样时间 避开封冻期。本研究采样时间为2015年6月18-21 日。高山微水体底栖动物使用一种适用于不同水体 环境的水体、生物、沉积物采集器进行采集(刘硕然 等, 2018)。采样器底面积 $0.06 \mathrm{~m}^{2}$, 在每个微水体离 岸距离 $0.5 \mathrm{~m}$ 处对微水体底泥和水样进行采集。将采 集到的底泥在500 $\mu \mathrm{m}$ 网眼(40目)的篎网中清洗; 再 将清洗后残余物中的生物手工挑拣至标本瓶，加入 $10 \%$ 甲醛溶液保存。高山溪流底栖动物使用索伯网 (定量面积 $0.09 \mathrm{~m}^{2}, 500 \mu \mathrm{m}$ 网眼, 40 目)进行采集, 每 个样点设 5 个重复, 将采集到的生物手工挑拣至标 本瓶，并保存于 $95 \%$ 的酒精中。将生物标本带回实 验室, 在光学显微镜(Olympus SZ61, 中国)下分类 计数, 尽可能鉴定到最低级的分类单元。

\section{3 环境因子测定}

在采集生物样品的同时, 采用便携式手持水质 多参数分析仪(YSI Professional Plus, 美国)测定水 环境的电导率(conductivity, Cond)、溶解氧含量(dissolved oxygen, DO)、pH值; 采用GPS位置记录仪 (Garmin eTrex20, 中国台湾) 获得采样点的坐标和 海拔 (altitude, Alt); 采用便携式手持浊度仪 (SGZ-200BS，中国)测定水体的浊度(turbidity, Turb)。 通过带刻度的金属长杆测定水深(depth)、底泥深度 (bottom sediment depth, BSD); 用经过微水体几何 中心的长轴和短轴长度的乘积估算获得微水体面 积(area)。采用流速仪(Global Water FP111, 美国)测 定溪流的流速(flow velocity, FV)和水深; 用皮尺测 量溪流的宽度(width)。

在采集生物样品的同时, 采集 $500 \mathrm{~mL}$ 水样装于 瓶中, 在采样现场加入浓硫酸, 调节水样 $\mathrm{pH}$ 值小于 2 并冷藏保存, 带回实验室进一步分析。总氮含量 (total nitrogen, TN) 通过碱性过硫酸钾消解紫外分光 光度法测定(GB11894-1989); 总磷含量(total phosphorus, TP) 通过钼酸铵分光光度法测定(GB118931989); 化学需氧量(chemical oxygen demand, COD) 通过重铬酸盐法测定(HJ 828-2017); 总有机碳含量 (total organic carbon, TOC)通过TOC分析仪(Shimadzu TOC-V CPH, 日本)测定。

\section{4 数据分析}

高山微水体及溪流生境优势分类单元和稀有
分类单元的鉴别通过分别绘制分类单元多度排序 曲线并人工寻找曲线拐点来确定。根据各个区域分 类单元的丰富度绘制微水体与溪流分类单元维恩 图，其饼图的大小与分类单元丰富度无关，仅显示 区域间共有分类单元和特有分类单元信息。使用软 件PC-ORD Version 7.04 (McCune \& Mefford, 2016) 计算物种多样性并制作群落分布热图(heatmap)。其 中，物种丰富度以 $S$ 表示，物种多样性用 Shannon-Wiener指数 $\left(H^{\prime}\right)$ 表示。功能多样性计算依据Poff 等(2006)的描述, 选取其中能够通过形态特征和野 外行为观察进行鉴别的 9 个功能性状(包括飞行能 力、游泳能力、吸附能力、保护壳、形状、呼吸方 式、个体大小、生活型、营养习性)并分别赋值; 然 后将各样点群落结构特征与功能多样性赋值列表 结合进行计算。在计算过程中, 功能性状赋值首先 标准化到0-1之间, 然后采用矩阵乘法与加权平均 相结合的方法计算功能多样性的群落加权平均 (community-weighted means, CWM)矩阵。再通过功 能多样性离差(functional dispersion, FDis)计算群落 的功能多样性, FDis体现了物种与加权质心(c)的加 权平均距离。FDis的计算公式(Laliberte \& Legendre, 2010):

$$
\begin{aligned}
& \text { FDis }=\frac{\sum a_{j} z_{j}}{\sum a_{j}} \\
& \mathrm{c}=\frac{\sum a_{j} x_{i j}}{\sum a_{j}}
\end{aligned}
$$

其中: $a_{j}$ 为 $j$ 物种的丰富度, $z_{j}$ 为 $j$ 物种到性状 $i$ 空间维 度上的加权质心 $\mathrm{c}$ 的距离, $x_{i j}$ 为 $j$ 种对性状 $i$ 的属性(由 功能多样性的 CWM距离矩阵获得)。FDis的计算通 过软件PC-ORD Version 7.04实现(McCune \& Mefford, 2016)。

采用软件SPSS Statistics Version 22完成高山微 水体与溪流之间水生生物多样性和环境数据的差 异分析。首先通过Kolmogorov-Smirnov检验(K-S检 验)检查数据是否为正态分布, 若数据符合正态分 布, 则用独立样本 $T$ 检验; 若数据不符合正态分布, 则用非参数检验(Kruskal-Wallis检验)分析数据集间 的差异。

通过主坐标分析(principal co-ordinates analysis, $\mathrm{PCoA})$ 对环境因子的空间分布格局进行分析。环境 因子PCoA计算时排除了海拔，以降低采样点的选 
择对排序的影响。PCoA各样点间的距离以欧式距 离计算(Borcard \& Legendre, 2002), 变量的计算值 经过加权平均法处理, 双标图中不显示 $r^{2}<0.2$ 的环 境因子, 并以最小凸多边形框定各分组。环境因子 与生物群落的相关分析首先通过去趋势对应分析 (detrended correspondence analysis, DCA)计算得第1 轴长度为 4.99 , 判别生物群落属单峰模型, 选用典 范对应分析(canonical correspondence analysis, CCA)。 在CCA分析过程中, 未对环境因子进行篮选, 双标 图中不显示 $r^{2}<0.2$ 的环境因子, 环境变量经Z值标 准化处理, 并以最小凸多边形框定各分组, 因area 和width的单位不同, 在微水体和溪流之间无法比 较，故CCA分析剔除这两个环境变量。PCoA、DCA、 CCA运用软件PC-ORD Version 7.04 (McCune \& Mefford, 2016)完成。

\section{2 结果}

\section{1 环境因子}

除化学需氧量外, 其他表征水体营养状态的水 化学指标在溪流和微水体之间的差异均不显著, 而 表征水体物理属性的很多指标则存在显著差异(表
1)。具体体现为: 电导率、溶解氧、 $\mathrm{pH}$ 值在溪流和 微水体之间的差异极显著 $(P<0.01)$, 水深、化学需 氧量在溪流和微水体之间的差异显著 $(P<0.05)$; 而 海拔、浊度、总氮、总磷和总有机碳在溪流和微水 体之间的差异不显著 $(P>0.05)$ 。因微水体面积、底 泥深度、溪流宽和流速在微水体与溪流生境之间没 有可比性, 故未进行比较。各样点环境因子的PCoA 排序(图2)显示, 环境因子在微水体和溪流之间呈现 非常明显的差别。相比溪流生境，微水体生境组成 的最小凸多边形面积较大, 这说明微水体的环境异 质性较大。另外, 结合表1和图2可知，电导率、水深 和 $\mathrm{pH}$ 是溪流与微水体环境异质性的主要影响因子。

\section{2 底栖动物特征}

共鉴定出底栖动物 57 个分类单元，涵盖了 12 个 目、 25 个科、 40 个属(附录1)。其中，高山微水体共 鉴定出 30 个分类单元，总计 1,821 头。个体数超过 80 头的优势分类单元6个(图3A), 分别是：冲绳摇蚊 Chironomus okinawanus (661头)、Chironomus javanus (479头)、Chironomus circumdatus (267头)、

Psectrocladius obvius (107头)、水丝蚓属一种 Limnodrilus sp. (96头)和摇蚊属一种 Chironomus sp.

表1 溪流与微水体各环境因子描述及差异性分析

Table 1 List of the descriptive statistics and difference in environmental variables between stream and micro-waterbody

\begin{tabular}{|c|c|c|c|c|c|c|}
\hline \multirow{2}{*}{$\begin{array}{l}\text { 环境因子 } \\
\text { Environmental variables }\end{array}$} & \multicolumn{3}{|c|}{ 微水体 Micro-waterbody } & \multicolumn{3}{|c|}{ 溪流 Stream } \\
\hline & $\begin{array}{l}\text { 最小值 } \\
\text { Min. }\end{array}$ & $\begin{array}{l}\text { 最大值 } \\
\text { Max. }\end{array}$ & $\begin{array}{l}\text { 平均值 } \pm \text { 标准差 } \\
\text { Mean } \pm \mathrm{SD}\end{array}$ & $\begin{array}{l}\text { 最小值 } \\
\text { Min. }\end{array}$ & $\begin{array}{l}\text { 最大值 } \\
\text { Max. }\end{array}$ & $\begin{array}{l}\text { 平均值 } \pm \text { 标准差 } \\
\text { Mean } \pm \mathrm{SD}\end{array}$ \\
\hline 海拔 Alt (m) ${ }^{\mathrm{ns} 2}$ & $3,266.00$ & $3,332.00$ & $3,299.70 \pm 27.34$ & $3,087.00$ & $3,586.00$ & $3,364.90 \pm 188.82$ \\
\hline 电导率 Cond $(\mu \mathrm{s} / \mathrm{cm})^{* * 2}$ & 2.94 & 10.45 & $5.12 \pm 1.33$ & 10.82 & 34.50 & $17.54 \pm 8.84$ \\
\hline 溶解氧含量 DO (mg/L) $)^{* * 1}$ & 3.13 & 7.51 & $5.68 \pm 0.92$ & 6.22 & 7.18 & $6.72 \pm 0.27$ \\
\hline $\mathrm{pH}$ 值 $\mathrm{pH}^{* * 1}$ & 5.48 & 6.20 & $5.84 \pm 0.24$ & 5.80 & 6.55 & $6.23 \pm 0.26$ \\
\hline 面积 Area $\left(\mathrm{m}^{2}\right)^{\text {none }}$ & 3.00 & 160.00 & $26.15 \pm 33.65$ & I & / & l \\
\hline 水深 Depth $(\mathrm{cm})^{* 1}$ & 8.00 & 43.00 & $27.70 \pm 10.09$ & 6.00 & 26.00 & $18.33 \pm 6.48$ \\
\hline 底泥深度 BSD (cm) ${ }^{\text {none }}$ & 9.50 & 58.00 & $26.09 \pm 13.02$ & I & I & I \\
\hline 浊度 Turb (NTU) $)^{\mathrm{ns} 1}$ & 0.00 & 3.70 & $1.42 \pm 1.08$ & 0.00 & 2.40 & $0.98 \pm 1.06$ \\
\hline 总氮 TN (mg/L) ${ }^{\mathrm{ns} 1}$ & 0.096 & 0.729 & $0.300 \pm 0.170$ & 0.115 & 0.445 & $0.280 \pm 0.110$ \\
\hline 总磷 TP (mg/L) ${ }^{\mathrm{ns} 1}$ & 0.024 & 0.104 & $0.046 \pm 0.016$ & 0.036 & 0.068 & $0.050 \pm 0.010$ \\
\hline 化学需氧量 COD (mg/L) ${ }^{* 1}$ & 0.160 & 4.730 & $2.150 \pm 1.290$ & 0.160 & 5.056 & $3.320 \pm 1.420$ \\
\hline 总有机碳含量 TOC $(m g / L){ }^{\mathrm{ns} 1}$ & 0.924 & 7.590 & $4.270 \pm 1.640$ & 3.043 & 6.963 & $4.800 \pm 1.070$ \\
\hline 溪流宽度 Width (m) ${ }^{\text {none }}$ & I & I & I & 0.50 & 5.30 & $2.53 \pm 1.78$ \\
\hline 溪流流速 $\mathrm{FV}(\mathrm{m} / \mathrm{s})^{\text {none }}$ & l & I & l & 0.16 & 0.93 & $0.58 \pm 0.31$ \\
\hline
\end{tabular}

以独立样本 $T$ 检验(上标为 1 )和非参数检验(上标为 2 )比较微水体与溪流之间环境因子的差异。 $* P<0.05 ; * * P<0.01$; ns表示差异不显著; none 表示无可比性, 不进行分析。

The difference of each environmental variable between micro-waterbody and stream are tested using independent sample $T$ test (with superscript 1 ) or nonparametric tests (with superscript 2). $* P<0.05$; ** $P<0.01$; ns indicates non-significant; none means do not perform the analysis because the data sets are not comparable. 


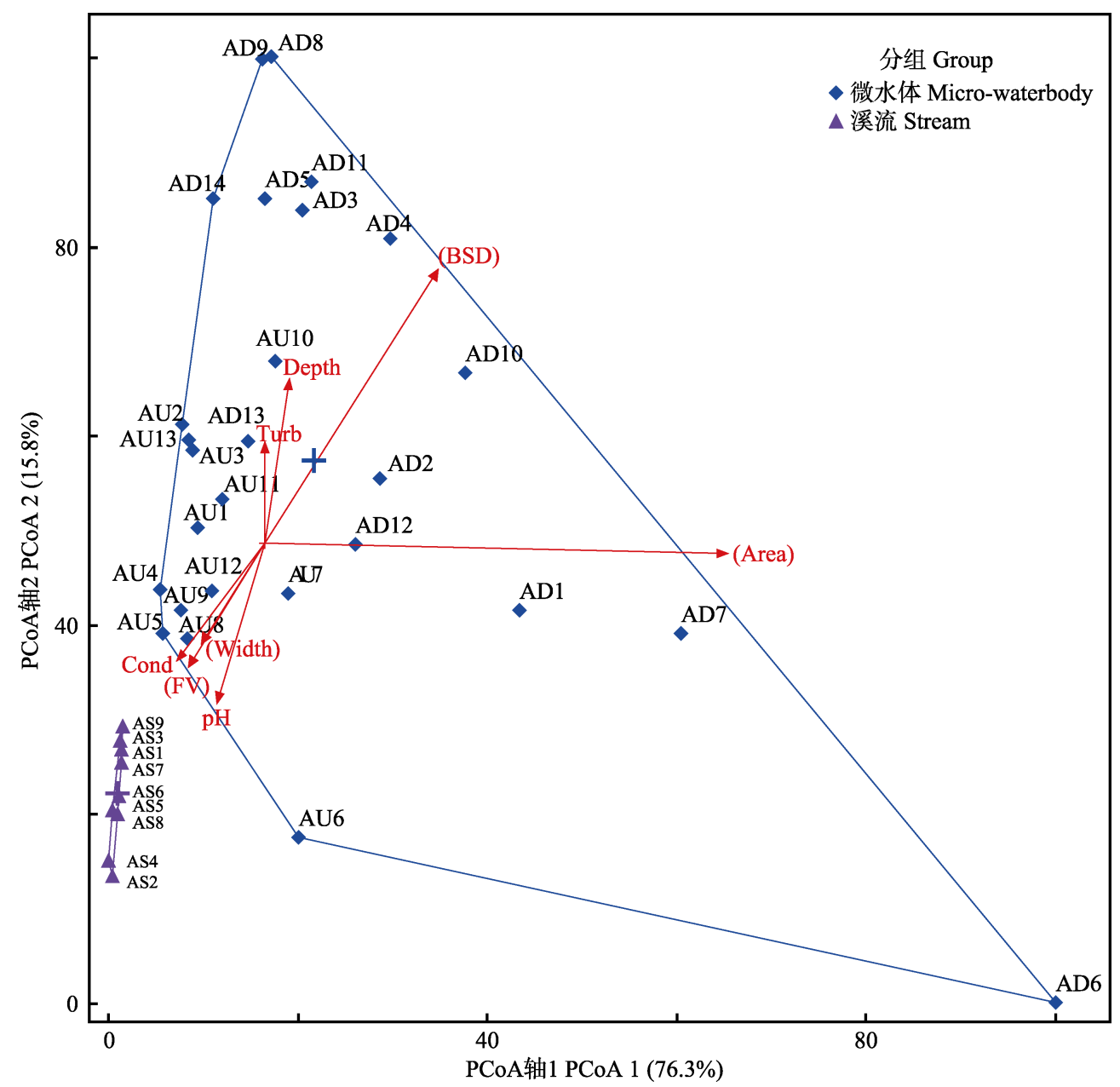

图2 基于环境因子的PCoA双标图。AS为溪流样点, AU、AD为微水体样点。Depth: 水深; Area: 面积; Width: 溪流宽度; FV: 流速; Cond: 电导率; Turb: 浊度。

Fig. 2 Biplot of PCoA calculated based on environmental factors. AS indicates stream sites, AU and AD indicate micro-waterbody sites. Depth, Water depth; Area, Waterbody surface area; Width, Stream width; FV, Flow velocity; Cond, Conductivity; Turb, Turbidity.

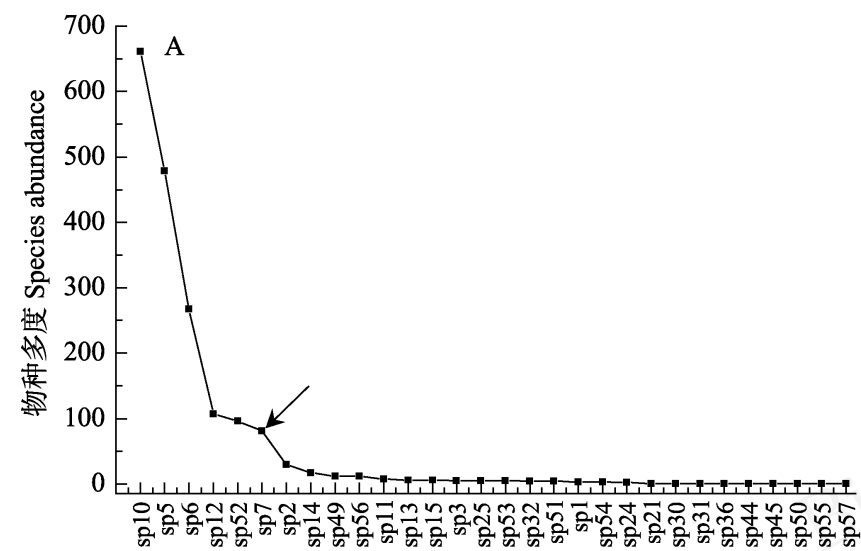

物种 Species

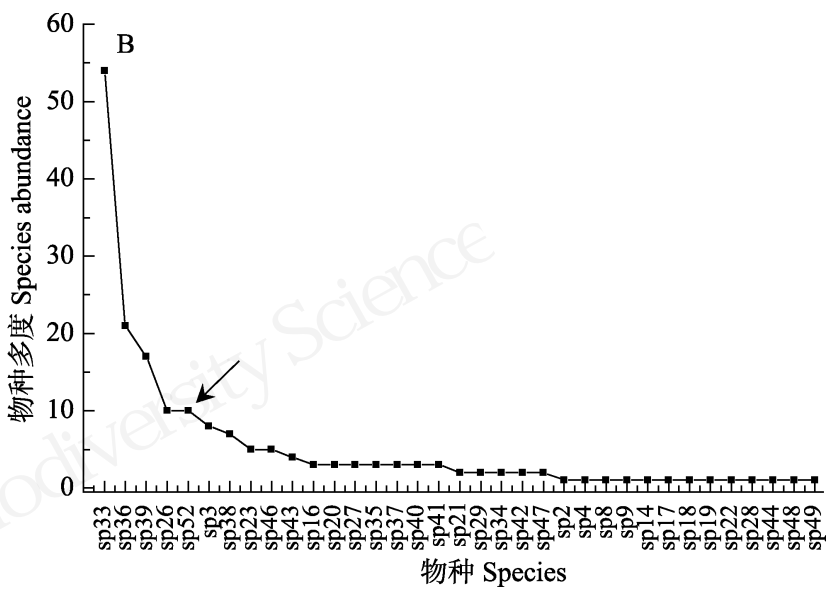

图3 高山微水体 $(\mathrm{A})$ 及溪流 $(\mathrm{B})$ 分类单元多度排序曲线(箭头所示为曲线拐点, 作为优势分类单元与稀有分类单元分界)，横 坐标为物种排列顺序, 对应附录1。

Fig. 3 Species abundance ranking curves for alpine micro-waterbody (A) and stream (B). Arrows indicate the inflection point of the curve between the dominant taxa and rare taxa. $\mathrm{X}$ axis refers to the species order in Appendix 1. 
(81头); 其余分类单元平均个体数为 5.4 头, 最多为 31 头。高山溪流共鉴定出 35 个分类单元，总计 185 头。个体数超过 10 头的优势分类单元 5 个(图3B), 分 别是: 四节蜉属一种(Beatis sp.1) (54头)、溪垓蜉属 一种(Rhithrogena sp.) (21头)、Amphinemura coreana (17头)、Optioservus sp. (10头)和水丝蚓属一种 (Limnodrilus sp.) (10头); 其余分类单元平均个体数 为 2.4 头, 最多为 8 头。底栖动物在溪流和微水体的 分布具有优势物种数量少但个体数多的特征, 即少 数分类单元个体数之和占总个体数比例较高。如上 述6个微水体优势分类单元占微水体总分类单元的 $20 \%$, 而个体数之和占微水体总个体数的 $92.9 \%$; 上述 5 个溪流优势分类单元占溪流总分类单元的 $14.3 \%$, 而个体数之和占溪流总个体数的 $60.5 \%$ 。
高山溪流与微水体底栖动物群落分布定性热 图(图4)表明: 大部分分类单元属于稀有分类单元， 且具有局地样点特有的特征。高山溪流与高山微水 体底栖动物分类单元丰富度维恩图(图5)结果表明: 溪流与微水体底栖动物群落分类单元的组成差异 较大。其中, 溪流特有分类单元有 27 个; 微水体特 有分类单元有 22 个; 溪流与微水体共有分类单元为 8 个, 分别是: Larsia atrocincta、长足摇蚊属一种 (Tanypus sp.)、巴瓦特维摇蚊(Tvetenia bavarica)、双 翅目一种(Diptera sp.)、溪垓蜉属一种(Rhithrogena sp.)、褐纹石蛾属一种(Eubasilissa sp.)、仰泳蝽属一 种(Notonecta sp.)和水丝蚓属一种(Limnodrilus sp.)。

2.3 底栖动物群落结构特征及环境驱动因子

底栖动物群落物种丰富度 $(S)$ 在溪流与微水体

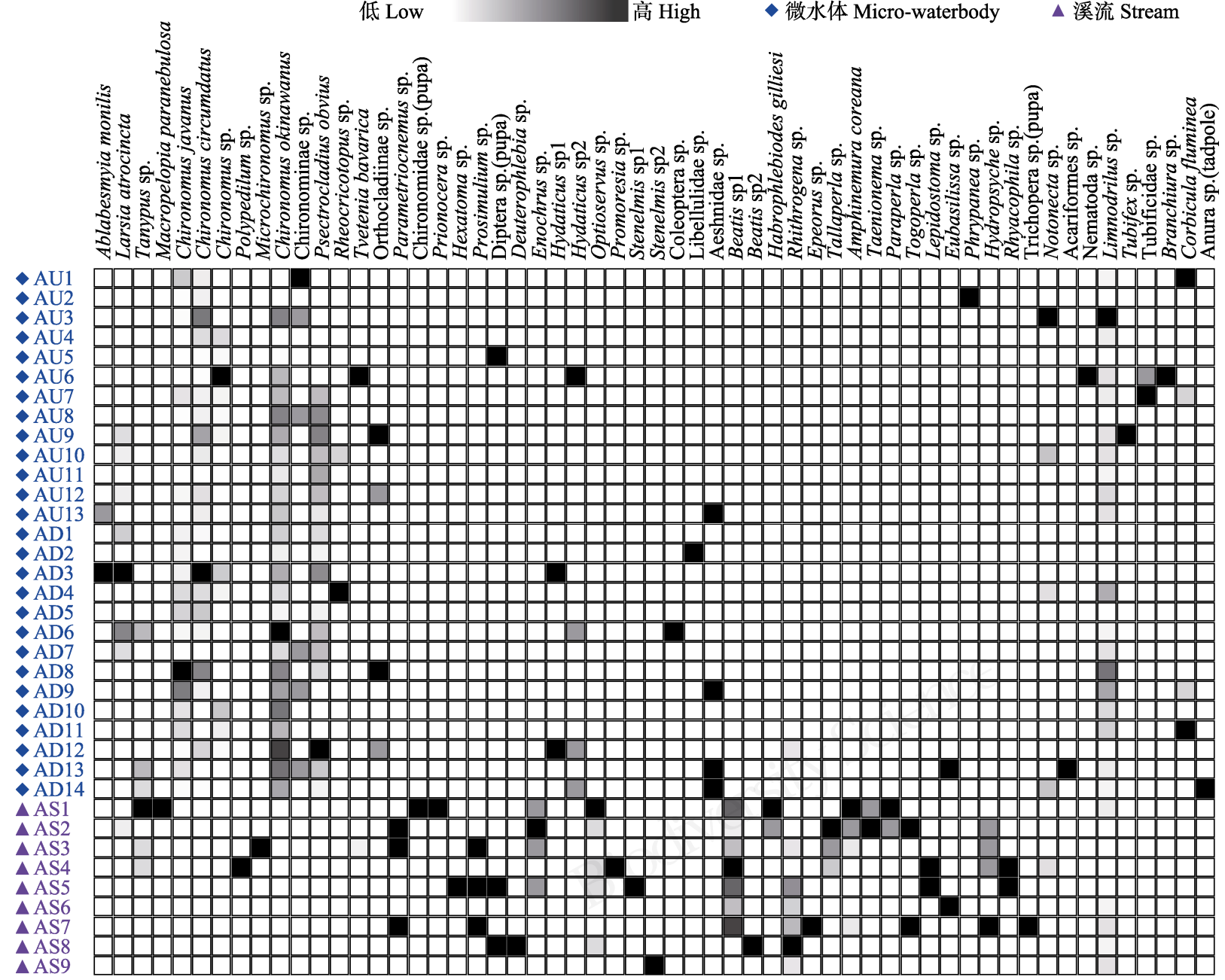

图4 底栖动物物种在样点间分布热图(分类单元物种多度以颜色深浅区分)

Fig. 4 Heatmap of the benthic species distribution among the collection sites. The species abundance for each taxon marked by the color shade 


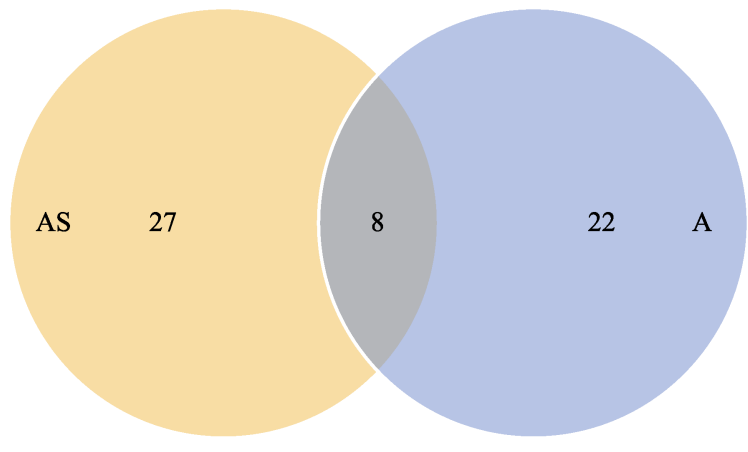

图5 溪流与微水体分类单元丰富度维恩图

Fig. 5 The Venn diagram between the micro-waterbody and stream by taxon richness

表2 溪流与微水体底栖动物群落的物种丰富度 $(S)$ 、物种多 样性 $\left(H^{\prime}\right)$ 和功能多样性(FDis)

Table 2 List of the species richness, species diversity and functional diversity between stream and micro-waterbody

\begin{tabular}{|c|c|c|c|c|c|c|}
\hline & \multicolumn{3}{|c|}{ 微水体 Micro-waterbody } & \multicolumn{3}{|c|}{ 溪流 Stream } \\
\hline & $\begin{array}{l}\text { 最小 } \\
\text { 值 } \\
\text { Min. }\end{array}$ & $\begin{array}{l}\text { 最大 } \\
\text { 值 } \\
\text { Max. }\end{array}$ & $\begin{array}{l}\text { 平均值 } \pm \\
\text { 标准差 } \\
\text { Mean } \pm \text { SD }\end{array}$ & $\begin{array}{l}\text { 最小 } \\
\text { 值 } \\
\text { Min. }\end{array}$ & $\begin{array}{l}\text { 最大 } \\
\text { 值 } \\
\text { Max. }\end{array}$ & $\begin{array}{l}\text { 平均值 } \pm \\
\text { 标准差 } \\
\text { Mean } \pm \text { SD }\end{array}$ \\
\hline$S^{\text {ns1 }}$ & 3.00 & 10.00 & $6.26 \pm 2.07$ & 3.00 & 12.00 & $8.67 \pm 3.43$ \\
\hline$H^{\prime{ }^{* 1}}$ & 0.882 & 1.649 & $1.240 \pm 0.250$ & 1.011 & 2.265 & $1.720 \pm 0.460$ \\
\hline FDis $^{* * 1}$ & 0.000 & 0.469 & $0.220 \pm 0.130$ & 0.544 & 0.968 & $0.820 \pm 0.130$ \\
\hline
\end{tabular}

以独立样本 $T$ 检验 (上标为 1 )比较微水体和溪流之间多样性的差异。* $P<0.05 ; * * P<0.01$; ns表示差异不显著。

The difference of diversity between stream and micro-waterbody are tested using independent sample $T$ test (with superscript 1 ). ${ }^{*} P<0.05$; ** $P<0.01$; ns indicates non-significant.

之间的差异不显著 $(P>0.05)$, 不过溪流平均物种丰 富度高于微水体。物种多样性 $\left(H^{\prime}\right)$ 在溪流与微水体 之间的差异显著 $(P<0.05)$, 溪流物种多样性平均值 显著高于微水体。功能多样性(FDis)体现出与物种 多样性类似的趋势, 溪流与微水体之间的差异极显 著 $(P<0.01)$, 且溪流生境FDis的最小值大于微水体 生境的最大值(表2)。CCA排序结果显示: AS区和A 区底栖动物群落在CCA排序图上完全不重叠(图6)。 这表明溪流生境与微水体生境底栖动物群落结构 表现出非常明显的差异。影响溪流和微水体之间底 栖动物群落产生差别的主要环境因子为电导率和 $\mathrm{pH}$ 值, 而影响溪流群落内部产生差别的主要环境 因子为海拔和流速, 微水体内部群落的差异与环境 因子关系不大。

\section{3 讨论}

\section{1 高山微水体与溪流环境因子差异}

尽管本研究区域内的大部分微水体串联系统
中水流最终汇集并流入溪流，与溪流形成连通，但 微水体仍保持着特有的生境类型。从环境因子特征 来看, 溪流与微水体在环境指标上产生的差异主要 是由生境固有的物理性质决定的。微水体的特征与 湖泊类似，比溪流拥有相对较大的敞水面积、较深 的水深、相对静止的水文特征、富集了较厚的底泥 沉积物。而溪流则有相对较大的流量、较浅的水深、 基本没有底泥沉积物的富集、敞水面为典型的狭长 条状。这样的生境特征差别势必造成溪流与微水体 某些水质指标的差异。对溪流而言，流速与海拔、 溶解氧和溪流宽度具有显著的相关性，因此，由于 局地地形因素导致的流速差异可能是溪流生境环 境异质性产生的主要因素之一。

\section{2 高山微水体与溪流底栖动物群落结构与多样 性差异及其驱动因子}

高山溪流与高山微水体属于两种截然不同的 生境类型，底栖动物因长期适应，表现出对不同生 境类型极大的依赖性。如蜉蝣目、毛翅目和襀翅目 昆虫幼虫普遍利用溪流生境(Belmar et al, 2013), 而 摇蚊科、颤蚓科等的幼虫则多检出于较深的底质沉 积物生境(Real et al, 2000; Bazzanti et al, 2003)。唡开 究中底栖动物群落的分布也符合上述规律, 溪流与 微水体的底栖动物分类单元组成差异明显, 共有部 分比例很小, 特有分类单元丰富(图5)。可见, 生境 类型的差异是导致本研究中底栖动物群落产生差 异的主要原因。

从生物多样性的角度分析, 溪流底栖动物的物 种多样性和功能多样性均高于微水体并存在显著 差异(表2)。就溪流而言, 底栖动物物种多样性与海 拔、溪流宽度、流速、溶解氧等环境因子显著相关。 因此，无论在不同生境间还是在同一生境内，底栖 动物多样性的差异可能都是由于生境特征的差异 而导致的(Bonada et al, 2007; Bogan et al, 2013)。

\section{3 高山微水体和高山溪流对生物多样性的维持 作用}

从区域尺度上看，高山溪流沿海拔梯度形成的 环境因子变化是导致溪流生物群落差异的主要原 因(Miserendino, 2001; Henriques-Oliveira \& Nessimian, 2010), 而与溪流流速相关的环境因子则影响 局地生物群落的格局(Chakona et al, 2008)。

本研究区域地理范围较小, 高山溪流总长度不 超过 $3 \mathrm{~km}$, 高山微水体群总面积不超过 $0.2 \mathrm{~km}^{2}$, 总 


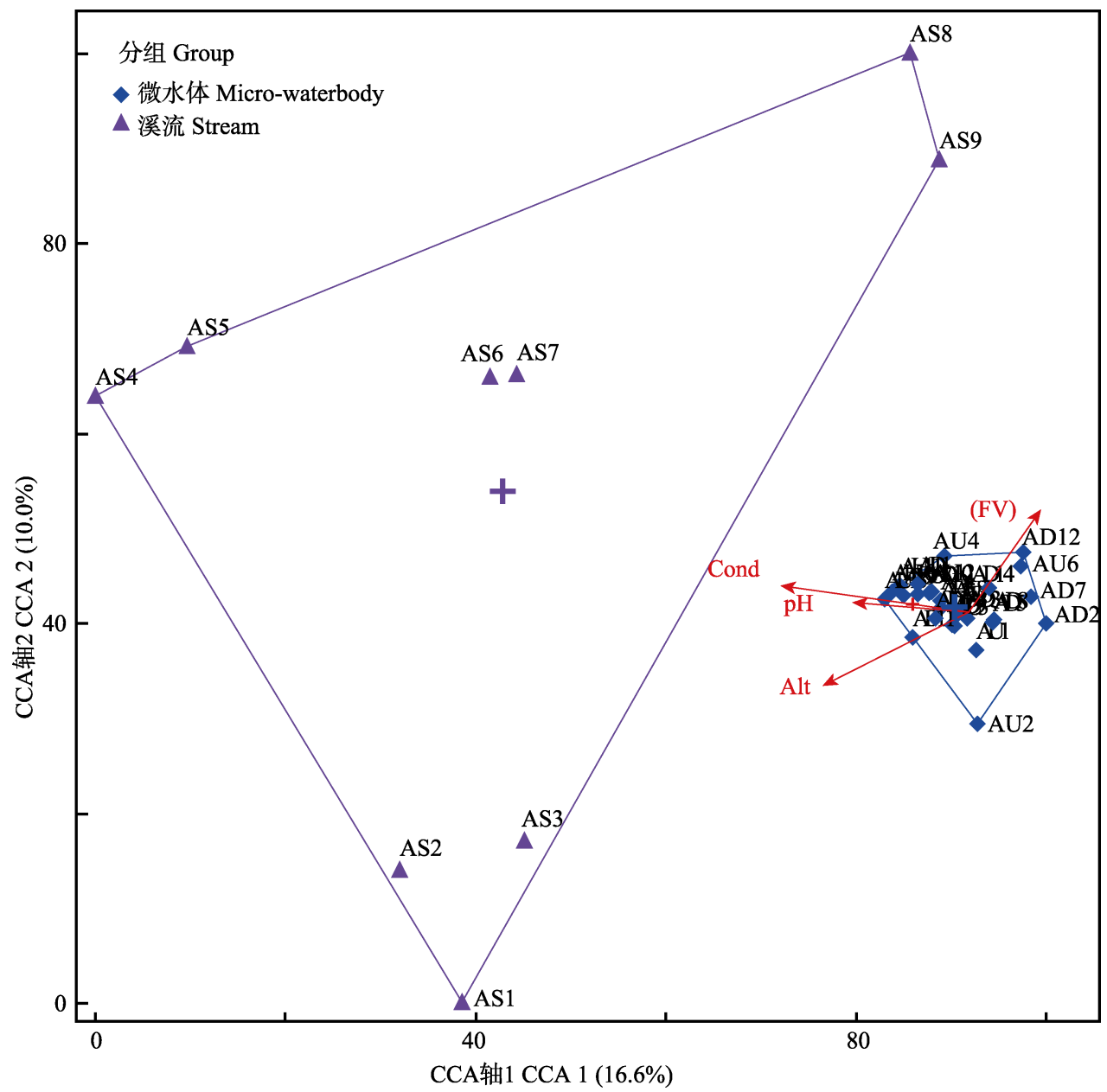

图6 基于底栖动物群落数据和环境因子的CCA双标图。AS为溪流样点, AU、AD为微水体样点。Alt: 海拔; FV: 流速; Cond: 电导率。

Fig. 6 Biplot of CCA which calculated based on the benthic community data and environmental factors. hydrogen ion concentration $(\mathrm{pH})$, flow velocity (FV) and conductivity (Cond). AS indicates stream sites, AU and AD indicate micro-waterbody sites. Alt, Altitude; FV, Flow velocity; Cond, Conductivity.

体海拔落差约500 m。如此小的地理区域内却产生 了丰富的生物多样性, 这也佐证了本研究区域为全 球生物多样性热点地区, 未来应加强对高山微水体 和高山溪流生物多样性不同维持机制的研究。

高山溪流与高山微水体拥有独特而丰富的底 栖动物类群，且一般位于流域上游，对区域生态系 统生物多样性的维持和物种库的形成具有重要意 义(Biggs et al, 2005; Scheffer et al, 2006)。对于高山 微水体, 虽然在微小尺度下的环境异质性很高, 但 生物群落的形成并不与环境因子相关, 可能存在其 他机制影响生物群落的构建过程, 如物种的扩散过 程(Leibold et al, 2004), 这对后续群落动态的研究 提供参考。无论从环境因子、底栖动物群落结构还 是从生物多样性的角度来分析, 高山溪流与微水体
均有明显的区别, 各自维持着独特的生态功能, 故 在未来的保护措施制定和科研立项工作中，应加以 重视。

\section{参考文献}

Altermatt F (2013) Diversity in riverine metacommunities: A network perspective. Aquatic Ecology, 47, 365-377.

Bazzanti M, Bella VD, Seminara M (2003) Factors affecting macroinvertebrate communities in astatic ponds in central Italy. Journal of Freshwater Ecology, 18, 537-548.

Belletti B, Rinaldi M, Buijse AD, Gurnell AM, Mosselman E (2015) A review of assessment methods for river hydromorphology. Environmental Earth Sciences, 73, 2079-2100.

Belmar O, Velasco J, Gutierrezcanovas C, Melladodiaz A, Millan A, Wood PJ (2013) The influence of natural flow regimes on macroinvertebrate assemblages in a semiarid Mediterranean basin. Ecohydrology, 6, 363-379. 
Biggs J, Williams P, Whitfield M, Nicolet P, Weatherby AJ (2005) 15 years of pond assessment in Britain: Results and lessons learned from the work of pond conservation. Aquatic Conservation-Marine and Freshwater Ecosystems, 15, 693-714.

Bogan MT, Boersma KS, Lytle DA (2013) Flow intermittency alters longitudinal patterns of invertebrate diversity and assemblage composition in an arid-land stream network. Freshwater Biology, 58, 1016-1028.

Bonada N, Rieradevall M, Prat N (2007) Macroinvertebrate community structure and biological traits related to flow permanence in a Mediterranean river network. Hydrobiologia, 589, 91-106.

Borcard D, Legendre P (2002) All-scale spatial analysis of ecological data by means of principal coordinates of neighbour matrices. Ecological Modelling, 153, 51-68.

Carrara F, Altermatt F, Rodrigueziturbe I, Rinaldo A (2012) Dendritic connectivity controls biodiversity patterns in experimental metacommunities. Proceedings of the National Academy of Sciences, USA, 109, 5761-5766.

Carrara F, Rinaldo A, Giometto A, Altermatt F (2014) Complex interaction of dendritic connectivity and hierarchical patch size on biodiversity in river-like landscapes. The American Naturalist, 183, 13-25.

Chakona A, Phiri C, Magadza CH, Brendonck L (2008) The influence of habitat structure and flow permanence on macroinvertebrate assemblages in temporary rivers in northwestern Zimbabwe. Hydrobiologia, 607, 199-209.

Dudgeon D, Arthington AH, Gessner MO, Kawabata ZI, Knowler D, Leveque C, Naiman RJ, Prieur-Richard AH, Soto D, Stiassny MLJ, Sullivan CA (2006) Freshwater biodiversity: Importance, threats, status and conservation challenges. Biological Reviews, 81, 163-182.

Henriques-Oliveira AL, Nessimian JL (2010) Aquatic macroinvertebrate diversity and composition in streams along an altitudinal gradient in southeastern Brazil. Biota Neotropica, 10, 115-128.

Hoffman M, Koenig K, Bunting G, Costanza J, Kristen JM (2016) Biodiversity Hotspots (version 2016.1). http: //doi.org/10.5281/zenodo.3261807. (accessed on 2019-03-18)

Laliberte E, Legendre PA (2010) Distance-based framework for measuring functional diversity from multiple traits. Ecology, 91, 299-305.

Leibold MA, Holyoak M, Mouquet N, Amarasekare P, Chase JM, Hoopes MF, Holt RD, Shurin JB, Tilman D, Loreau M, Gonzalez A (2004) The metacommunity concept: A framework for multi-scale community ecology. Ecology Letters, 7, 601-613.

Liu SR, He XY, Yang WS, Ren GP, Li YP, Zhou J, Cai QH, Xiao W (2017) Spatial distribution and significance of high mountain micro-waterbodies in northwestern Yunnan, China. Journal of Hydroecology, 38, 18-23. (in Chinese with English abstract) [刘硕然, 和晓阳, 杨文书, 任国鹏,
李延鹏, 周俊, 蔡庆华, 肖文 (2017) 滇西北高山微水体 空间分布格局及研究意义初探. 水生态学杂志, 38, 18-23.]

Liu SR, Li YP, Yan JG, Xiao W (2018) A Waterbody, Organisms and Sediment Sampling Equipment Used for Different Kinds of Water Environments. Chinese Patent. ZL201510837357.X. 2018-10-19. (in Chinese) [刘硕然, 李 延鹏，问家国，肖文 (2018) 一种适用于不同水体环境的 水体、生物、沉积物采集器. 中国专利: ZL201510837357. X. 2018-10-19.]

Liu SR, Lu T, Yang DD, Ren GP, He XY, Yang WS, Cai QH, Xiao W (2018) Spatiotemporal environmental heterogeneity of alpine micro-waterbodies. Fresenius Environmental Bulletin, 27, 8088-8095.

McCune B, Mefford MJ (2016) PC-ORD Multivariate Analysis of Ecological Data, Version 7.04. MjM Software, Gleneden Beach, Oregon.

Ministry of Ecology and Environment of the People's Republic of China (2017) HJ 828-2017. Water Quality Determination of Chemical Oxygen Demand: Dichromate Method. China Environmental Press, Beijing. (in Chinese with) [中华人民 共和国生态环境部 (2017) HJ 828-2017 水质化学需氧量 的测定: 重铬酸盐法. 中国环境出版社, 北京.]

Miserendino ML (2001) Macroinvertebrate assemblages in Andean Patagonian rivers and streams: Environmental relationships. Hydrobiologia, 444, 147-158.

Myers N (1988) Threatened biotas: Hot spots in tropical forests. The Environmentalist, 8, 187-208.

Myers N, Mittermeier RA, Mittermeier CG, Fonseca GA, Kent J (2000) Biodiversity hotspots for conservation priorities. Nature, 403, 853-858.

National Environmental Protection Administration (1990a) GB 11893-1989. Water Quality Determination of Total Phosphorus: Ammonium Molybdate Spectrophotometric Method. China Standard Press, Beijing. (in Chinese with) [国家环境 保护总局 (1990) GB 11893-1989 水质总磷的测定: 钼酸 铵分光光度法. 中国标准出版社, 北京.]

National Environmental Protection Administration (1990b) GB 11894-1989. Water Quality Determination of Total Nitrogen: Alkaline Potassium Persulfate Digestion UV Spectrophotometric Method. China Standard Press, Beijing. (in Chinese with) [国家环境保护总局 (1990) GB 11894-1989 水质总氮的测定: 碱性过硫酸钾消解紫外分光光度法. 中国标准出版社, 北京.]

Poff NL, Olden JD, Vieira NK, Finn DS, Simmons MP, Kondratieff BC (2006) Functional trait niches of North American lotic insects: Traits-based ecological applications in light of phylogenetic relationships. Journal of the North American Benthological Society, 25, 730-755.

Real M, Rieradevall M, Prat N (2000) Chironomus species (Diptera: Chironomidae) in the profundal benthos of Spanish reservoirs and lakes: Factors affecting distribution patterns. Freshwater Biology, 43, 1-18. 
Sala OE, Chapin FS, Armesto JJ, Berlow EL, Bloomfield J, Dirzo R, Huber-Sanwald E, Huenneke LF, Jackson RB, Kinzig AP, Leemans R, Lodge DM, Mooney HA, Oesterheld M, Poff NL, Sykes MT, Walker BH, Walker M, Wall DH (2000) Global biodiversity scenarios for the year 2100. Science, 287, 1770-1774.

Scheffer M, Van Geest GJ, Zimmer KD, Jeppesen E, Sondergaard M, Butler MG, Hanson MA, Declerck S, De Meester L (2006) Small habitat size and isolation can promote species richness: Second-order effects on biodiversity in shallow lakes and ponds. Oikos, 112, 227-231.

Stokstad E (2014) EPA science report signals start of wetlands battle. Science, 343, 15.

Taft B, Koncelik JP (2006) Methods for Assessing Habitat in Flowing Waters: Using the Qualitative Habitat Evaluation Index (QHEI). Division of Surface Water, Ohio EPA. http://epa.ohio.gov/portals/35/documents/QHEIManualJune 2006.pdf. (accessed on 2019-05-07)

(责任编委: 王洪铸 责任编辑: 时意专)

\section{附录 Supplementary Material}

\section{附录1 各分类单元鉴定结果及其多度列表}

Appendix 1 List of taxa from identification results and the abundance http://www.biodiversity-science.net/fileup/PDF/2019157-1.pdf

附录2 各样点物种丰富度、物种多度、物种多样性及功能多样性列表

Appendix 2 Species richness, species abundance, species diversity and functional diversity of each site http://www.biodiversity-science.net/fileup/PDF/2019157-2.pdf 